Ana Holck

Ritmo e Caos

\title{
Temporalidades Urbanas nas Obras de Piet Mondrian e Richard Serra
}

\section{DISSERTAÇÃO DE MESTRADO}

\section{DEPARTAMENTO DE HISTÓRIA \\ Programa de Pós-Graduação em História Social da Cultura}

Rio de Janeiro

Agosto de 2003 


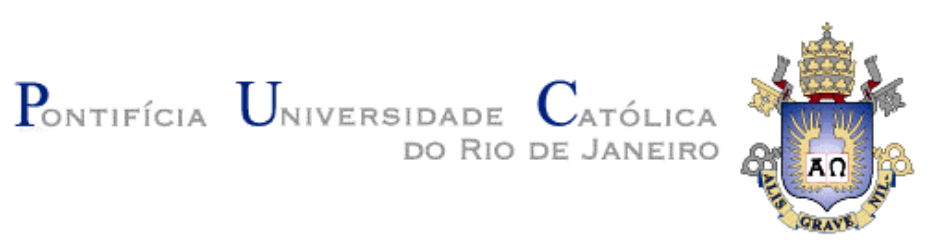

Ana Holck

\title{
Ritmo e Caos
}

Temporalidades Urbanas nas Obras de Piet Mondrian e Richard Serra

\begin{abstract}
Dissertação de Mestrado
Dissertação apresentada ao Programa de PósGraduação em História Social da Cultura do Departamento de História da PUC-Rio como parte dos requisitos parciais para a obtenção do título de Mestre em História
\end{abstract}

Orientador: Ronaldo Brito Fernandes

Rio de Janeiro Agosto de 2003 
Todos os direitos reservados. É proibida a reprodução total ou parcial do trabalho sem autorização da universidade, da autora e do orientador.

\section{Ana Holck}

Graduou-se em Arquitetura na FAU-UFRJ (Faculdade de Arquitetura e Urbanismo da Universidade Federal do Rio de Janeiro) em 2000. Em 2001 recebeu o Prêmio Paviflex em concurso nacional de arquitetura promovido pelo IAB-SP e em 2000 o Prêmio Arquiteto do Amanhã, concedido pelo IAB-RJ. Autora da resenha " $D o$ plano ao urbano", na revista Anima, ano II, n³, 2002, e da biografia, cronologia e bibliografia do livro "Iberê Camargo", de Paulo Venancio Filho, organizado e editado por Silvia Roesler e Instituto Cultural The Axis, Rio de Janeiro, 2001. É artista plástica e participou de exposições no Rio e em São Paulo.

Ficha Catalográfica

Holck, Ana

Ritmo e caos: temporalidades urbanas nas obras de Piet Mondrian e Richard Serra / Ana Holck; orientador: Ronaldo Brito Fernandes. - Rio de Janeiro : PUC, Departamento de História, 2003.

165 f. : il. ; $30 \mathrm{~cm}$

Dissertação (mestrado) - Pontifícia Universidade Católica do Rio de Janeiro, Departamento de História.

Inclui referências bibliográficas.

1. História - Teses. 2. Piet, Mondrian. 3. Serra, Richard. 4. Metrópole. 5. Música. 6. Dança. 7. Modernidade. 8. Europa. 9. Pósminimalismo. 10. Processo. 11. América. I. Fernandes, Ronaldo Brito. II. Pontifícia Universidade Católica do Rio de Janeiro. Departamento de História. III. Título. 


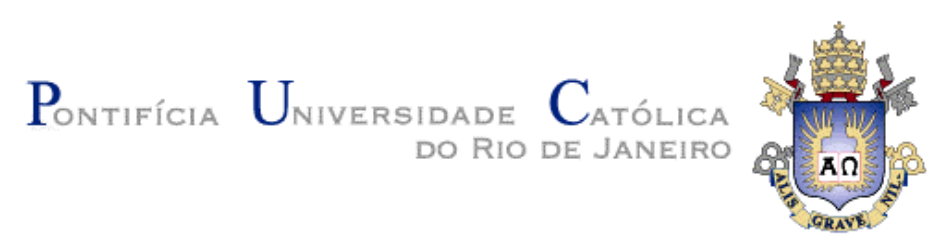

Ana Holck

\author{
Ritmo e Caos \\ Temporalidades Urbanas nas Obras de \\ Piet Mondrian e Richard Serra
}

Dissertação apresentada como requisito parcial para a obtenção do grau de Mestre pelo Programa de Pós-Graduação em História Social da Cultura do Departamento de História do Centro de Ciências Sociais da PUC-Rio. Aprovada pela Comissão Examinadora abaixo assinada.

Prof. Ronaldo Brito Fernandes
Orientador
Departamento de História - PUC-Rio

Prof. João Masao Kamita Departamento de História - PUC-Rio

Dr. Alberto de Almeida Prado Tassinari Departamento de Filosofia - FFLCH - USP

Prof $^{a}$ Dra $^{a}$. Zelia Milanez de Lossio e Seiblitz Vice-Decana de Pós-Graduação do Centro de Ciências Socais

PUC-Rio

Rio de Janeiro, 8 de agosto de 2003 


\section{Para Paulo Venancio Filho}




\section{Agradecimentos}

Ao Ronaldo Brito, meu singular orientador, pela generosidade e dedicação com que me conduziu neste percurso.

Ao CNPq e à PUC-Rio, pelos auxílios concedidos a este trabalho.

A Rose, Carlos Henrique e Guilherme, com amor, sem poder retribuir a paciência que tiveram comigo durante todos esses anos.

A Iole de Freitas, pelas conversas durante suas aulas no Parque Lage e a Regina Vaz e a Maria José Michalski, por terem cedo me despertado para o tema deste trabalho.

Aos professores José Thomaz Brum e João Masao Kamita, pelas valiosas contribuições feitas durante a defesa do projeto de dissertação.

A Christina Guido, pela revisão e a Juliana Samel, pela ajuda na tradução.

A Edna e a todo o pessoal do Departamento de História da PUC-Rio.

Aos colegas do Programa de Pós-Graduação em História Social da Cultura e aos amigos, Amalia Giacomini, Ana Paula Pontes, Bernardo Buarque de Holanda, Felipe Brandi, Gustavo Mendonça Prado, Laura Erber, Luisa Buarque de Holanda, Marisa Florido, Pedro Duarte Andrade e tantos outros que participaram deste 'processo'. 


\section{Resumo}

Holck, Ana; Fernandes, Ronaldo Brito. Ritmo e Caos: Temporalidades Urbanas nas Obras de Piet Mondrian e Richard Serra. Rio de Janeiro, 2003. 154 p. Dissertação de Mestrado - Departamento de História, Pontifícia Universidade Católica do Rio de Janeiro.

As obras de Piet Mondrian e Richard Serra possuem profunda relação com a metrópole. Seus trabalhos abrangeram distintas temporalidades urbanas no decorrer do século $\mathrm{XX}$, e foram permeados direta e indiretamente pela música e pela dança, artes temporais igualmente circunscritas ao fenômeno urbano. Mondrian identifica no ambiente moderno, dinâmico e veloz, da Paris da primeira metade do século XX, as premissas de sua teoria neoplástica do 'equilíbrio universal'. Procurava realizar sua teoria em outras artes além da pintura, de modo que ao integrar-se à vida, a arte desapareceria. Sua chegada a América coincide com a transferência do centro artístico mundial de Paris para Nova York. No rico embate entre Europa e América, percebemos as diferenças entre a monumental escala do Novo Continente e a permanente atividade de seu povo, em contraposição às dimensões tímidas e o temperamento contemplativo do homem do Velho Continente. Hoje, a mecanização das metrópoles já não é mais polêmica ou fascinante, mas responsável pela monótona e repetitiva vida urbana da qual partiu a obra dos Pós-Minimalistas, entre os quais, Richard Serra. O caráter utópico da pintura de Mondrian, cuja virtualidade se queria transformadora da realidade, visando a 'sociedade madura' exemplificada pela América, está ausente na arte do pós-guerra. Os planos inseridos por Serra no tecido urbano não querem sucumbir ao colapso do grid cubista, buscam o embate literal entre o habitante da metrópole e as coisas que o cercam, num mundo já muito pouco palpável.

\section{Palavras-chave}

Piet Mondrian, Richard Serra, Metrópole, Música, Dança, Modernidade, Urbanidade, Europa, Pós-Minimalismo, Processo, América. 


\section{Abstract}

Holck, Ana; Fernandes, Ronaldo Brito (Advisor). Rhythm and Chaos: Urban Temporalities In The Works of Piet Mondrian and Richard Serra. Rio de Janeiro, 2003. 154 p. MA Dissertation - Departamento de História, Pontifícia Universidade Católica do Rio de Janeiro.

The works of Piet Mondrian and Richard Serra are profoundly related to the metropolis. Their works embrace different urban temporalities elapsed in the twentieth century. They were directly and indirectly influenced by music and dance, temporal arts equally confined to the urban phenomenon. Mondrian identifies the 'universal balance' premises of his neoplastic theory in the modern, dynamic and fast Parisian environment of the first half of the twentieth century. He tries to accomplish his theory in other arts besides painting, so that by integrating art in life, the former would disappear. His arrival in America coincides with the artistic world center move from Paris to New York. In the cultural clash between Europe and America, one realizes the differences between the New World's monumental scale and its dynamic people, against the shy dimensions and contemplative nature of the men in the Old Continent. The metropolis' mechanization is no longer polemic or fascinating, but responsible for the monotonous and repetitive urban life, from which the Post-Minimalists emerged, among them Serra. The utopian character of Mondrian's painting, whose virtuality intended to transform reality, like America's 'ripe society' example, is absent in post-war art. The planes inserted by Serra in the urban fabric don't intend to succumb to the cubist grid's collapse. They seek the literal clash between the metropolis citizen and what surrounds him, in a very little tangible world.

\section{Keywords}

Art, Twentieth Century, Metropolis, Music, Dance, Modernity, Urbanity, Europe, Post-Minimalism, Process, America. 


\section{Sumário}

1 | Introdução 01

2 | Mondrian| A Pulsação da Metrópole

2.1 | Utopia urbana e sonora: o equilíbrio impossível 04

2.2 | Ruído e máquina: o impacto da cidade real 20

2.3 | Frenesi, ritmos dançantes e ação: os anos 20 e 30

2.4 | Big Apple e boogie-woogie: o duplo impacto 47

3 | Serra | A Metrópole Desencantada

3.1 | Grid em colapso: amnésia urbana 69

3.2 | Processo: a dimensão temporal da experiência 82

3.3 | 'A rotina tem seus encantos' 102

3.4 | Peso e gravidade: desequilíbrio iminente 116

3.5 | Cidade infinita: monumento ao caos 129

4 | Conclusão 145

5 | Referências bibliográficas 147 


\section{Lista de figuras}

1. Piet Mondrian. Composição $n^{\circ}$ VI, Fachada Azul, 1914, óleo s/ tela, 95.2X67.5 p.04

2. Piet Mondrian. Composição oval com planos de cor 1,1914 , óleo s/ tela, $107.5 \times 79 \quad$ p.04

3. Piet Mondrian. Pier e Oceano, 1915, óleo s/ tela, 85X108 p.05

4. Piet Mondrian. Composição em linha, 1916-17, óleo s/ tela, 108X108 p.05

5. Atelier de Mondrian na 26, Rue du Départ, $1926 \quad$ p.06

6. Casas de frente para o Canal, Amsterdam, século XVII p.06

7. Vista aérea de Amsterdam, 1928 e Plano da Expansão de Van Eesteren, 1928-34 p.07

8. Mies Van der Rohe. Projeto para torre de escritórios em Berlin, $1921 \quad$ p.10

9. Mies Van der Rohe. Edificio, Lake Shore Drive, Chicago, 1948-51 p.10

10. Johannes Vermeer. Jovem mulher com bilha d'água, 1664-65, óleo s/ tela, 45.7X40.6 p.11

11. Vista aérea de Nova York e planta de $1924 \quad$ p.11

12. Pablo Picasso. Violino e Uvas, 1912, óleo s/ tela, 50.6X61 p.12

13. Piet Mondrian. Composição com linhas cinzas. $1918 . \quad$ p.14

14. Piet Mondrian Composição (Checkerboard, cores claras), 1919, óleo s/ tela p.14

15. Luigi Russolo (esquerda) acompanhado de um assistente, com os bruiteurs, c. 1920 p.20

16. Giacomo Balla.Movimento de um cão na coleira, 1911, óleo s/ tela, $90.8 \times 100 \quad$ p.22

17. Piet Mondrian. Macieira em Flor, 1912, óleo s/ tela, 78X106 p.25

18. Piet Mondrian. Composição IV; Fox-Trot A, 1929, óleo s/ tela, $78.2 \times 78.2 \quad$ p.35

19. Atelier de Mondrian na Rue du Départ, $1926 \quad$ p.41

20. Le Corbusier. Ville Savoye, Poissy, 1928-31 p.42

21. Jerome Robbins. Fancy Free (Leonard Bernstein) 1944. American Ballet Theatre p.46

22. Vista aérea de campos americanos ordenados segundo a Land Ordinance p.50

23. Piet Mondrian. Place de La Concorde, 1938-43, óleo s/ tela, 94X95 p.51

24. Piet Mondrian. Trafalgar Square, 1939-43, óleo s/ tela, $145.5 \times 120$

25. Piet Mondrian. Broadway Boogie-Woogie, 1942-43, óleo s/ tela, 127X127 p.52

26. Piet Mondrian. Composição, 1926. oleo s/ teladiagonal $113,5 \mathrm{~cm} \quad$ p.52

27. Gerrit Rietvelt. Casa Scröder. $1924 . \quad$ p.53

28. Times Square, Nova York, $1938 \quad$ p.53

29. Piet Mondrian. New York City, 1941-42, óleo s/ tela, 119X114 p.55

30. Piet Mondrian. Victory Boogie-Woogie, 1942-44, óleo s/ tela, 127X127 (eixo vert.179) p.55

31. Barnett Newman. Vir Heroicus Sublimis. 1950-51. oleo s/ tela, $242.2 \times 541.7 \quad$ p.60

32. Jackson Pollock. Authum Rhythm: number 30, 1950. óleo s/ tela, 266.7 X $525.8 \quad$ p.63

33. Último atelier de Mondrian, 15 Leste, Rua 59, Nova York, 1944

34. Barnett Newman.Who's Afraid of Red Yellow and Blue IV,1969-70, acr. s/ tela,274X604 p.68

35. Robert Rauschenberg. Bed, 1955. técnica mista, 191X80X20 p.76

36. Richard Serra. Splashing. Castelli Warehouse, Nova York, 1968, chumbo, 18” X $26^{\prime} \quad$ p.82

37. Richard Serra jogando chumbo, Castelli Warehouse, Nova York, 1969

38. Ad Reinhardt. Abstract Painting. 1963, oleo s/ tela, 152.4X152.4 p.85

39. Richard Serra. Instalação de Props. Guggenheim, Nova York, 1969. 
40. Richard Serra. Casting. 1969. chumbo. 4" X $25 \times 15^{\prime} \quad$ p.86

41. Richard Serra. Cuting Device: Base Plate Measure. 1969. materiais diversos. p.86

42. Richard Serra. Fotogramas do filme Hand Catching Lead. 1968.99

43. Frank Stella. Die Fahne Hoch!, 1959. esmalte s/ tela 121" X 73" p.105

44. Donald Judd. Sem Título, 1965, placas de alumínio e vidro, 86.4X410.2X86.4 p.105

45. Richard Serra. Tearing Lead From 1:00 to 1:47, 1968. chumbo, 10X10' p.116

46. Richard Serra. Thirty-five Feet of Lead Rolled Up. 1968. chumbo, aprox. 5X24" p.116

47. Richard Serra. One Ton Prop (House of Cards), 1969, chumbo p.117

48. Richard Serra. Stacked Steel Slabs (Skullcracker). California, 1969, aço, 20X8X10' p.119

49. Donald Judd. Sem Título, 1978, aço e alumínio anodizado, 9X40X31 in. cada p.119

50. Trisha Brown. Man Walking Down The Side of a Building. 1970 p.122

51. Trisha Brown. Walking on the Wall. Nova York, $1974 \quad$ p.122

52. Claes Oldenburg. Soft Washstand, 1965, vinil e estrutura metálica, 137X106X57 p.125

53. Richard Serra. Strike: To Roberta and Rudy. 1969-71. aço, 8 X 24' X 1" p.129

54. Richard Serra. Terminal. Bochum, Alemanha, $1977 \quad$ p.129

55. Trisha Brown. Roof Piece. Nova York, $1973 \quad$ p.134

56. Richard Serra. Saint John's Rottary Arc. Nova York, 1980 p.135

57. Richard Serra. Saint John's Rottary Arc. Nova York, 1980, vista aérea p.135

58. Richard Serra, T.W.U., 1980. aço cor-ten p.136

59. Richard Serra. Saint John's Rottary Arc. Nova York, 1980, vistas p.136

60. Robert Smithson. Spiral Jetty. Great Salt Lake, Utah, 1969-1970, vista aérea p.141

61. Richard Serra, Circuit, 1972. aço p.143

62. Richard Serra, Tilted Arc, 1981-89. aço-corten. Instalada na Federal-Plaza, Nova York p.145

\section{Lista de músicas}

1. Boogie Woogie Prayer. 2:03 (Albert Ammons, Meade 'Lux' Lewis, Pete Johnson). 1944.

2. Clapping Music. 4:48 (Steve Reich). 1972.

3. It's Gonna Rain. primeira parte 8:04 (Steve Reich). 1965 